\title{
Magneto-optic Three-Dimensional Holographic Display with Tilling Optical Addressing Method
}

\author{
Hiroyuki Takagi*, Kazuki Nakamura, Sotaro Tsuda, \\ Taichi Goto, Pang Boey Lim and Mitsuteru Inoue \\ Toyohashi University of Technology, 1-1 Hibarigaoka, \\ Tempaku, Toyohashi Aichi, 441-8580 Japan
}

(Received February 16, 2015; accepted June 1, 2015)

Key words: holography, thermomagnetic recording, amorphous magnetic materials, tiling optical addressing, three-dimensional (3D) display

A holographic display is a realistic three-dimensional (3D) display because it produces an exact copy of the wave front of scattered light from 3D objects. It demands a wide viewing angle for 3D visualization. However, the viewing angle of holographic displays based on conventional spatial light modulators (SLMs) is less than $3^{\circ}$. The pixel pitch of conventional SLMs is in the range of 10-100 $\mu \mathrm{m}$. Recently, we have fabricated a two-dimensional pixel array with sub-micrometer-scale pixels for a wide-viewingangle holographic display. The pixels were driven by a thermomagnetic recording system using a metal mask that formed fringe patterns. However, the reconstructions of $3 \mathrm{D}$ images were fixed. In this paper, we describe the results of the reconstruction of any $3 \mathrm{D}$ images using a tilling optical addressing method. The tilling optical method reproduced any magnetic fringe pattern with $5 \times 10^{7}$ pixels for the reconstruction of $3 \mathrm{D}$ images.

\section{Introduction}

A holographic display is expected to produce a realistic three-dimensional (3D) display without special glasses because it produces an exact copy of the wave front of scattered light from an object. ${ }^{(1-8)}$ In general, a 3D holographic display demands wide viewing angles for $3 \mathrm{D}$ visualization.

However, the viewing angle $\theta$ of holographic displays based on conventional spatial light modulators (SLMs) is less than $3^{\circ}$ in accordance with eq. (1): ${ }^{(9)}$

$$
\theta=\sin ^{-1}(\lambda / 2 p)
$$

*Corresponding author: e-mail: takagi@ee.tut.ac.jp 
where $\lambda$ is the wavelength of the reference light, and $p$ is the pixel pitch of the SLMs. The $p$ of conventional SLMs [liquid crystal devices (LCDs) ${ }^{(10)}$ and digital micromirror devices (DMDs) $\left.)^{(11)}\right]$ is in the range of $10-100 \mu \mathrm{m}$. Downsizing the pixel sizes of SLMs for a wide viewing angle is limited by the driving current lines and other limitations of the fabrication process. Therefore, we developed a holographic display with a wide viewing angle using magnetic pixels (3D-MOSLM). ${ }^{(12)}$ To form magnetic pixels, we used a thermomagnetic recording system ${ }^{(13-16)}$ to control the direction of magnetization by the optical addressing method. This method has the advantage that it is possible to fabricate sub-micrometer-scale pixel arrays without a driving line and a pixel structure on the magnetic medium.

We have reported that the 3D image can be reconstructed with a wide viewing angle from sub-micrometer-scale magnetic pixels. To write many magnetic submicron-size pixels on the array, a frame-written laser system was used; the beam from a pulsed laser passed a two-dimensional (2D) hologram metal pattern positioned by a three-axis positioning stage. The magnetic pixel was formed with dimensions of $800 \times 900 \mathrm{~nm}^{2}$, and the viewing angle of the reconstructed image was more than $30^{\circ}$. However, for demonstrating the principle, the reconstructed 3D images were fixed, because this system used a metal mask with a fixed 2D hologram pattern. Therefore, the display could not change the $3 \mathrm{D}$ images because the metal mask was used to write the hologram pattern.

In this paper, we describe the results of forming magnetic pixel patterns using the tilling optical addressing method. In this method, a part of the fringe images is shown on DMDs and transfers the fringe pattern to a magnetic film. This method can enlarge the area of a $2 \mathrm{D}$ hologram pattern when the writing position is adjusted by an $x-y-z$ stage.

\section{Experiments}

First, the magnetic film was an amorphous $\mathrm{Tb}_{78} \mathrm{Fe}_{22}(a-\mathrm{TbFe})$ film (100 nm thick) with perpendicular magnetization ${ }^{(17-19)}$ fabricated on a glass substrate by ion beam sputtering. The magnetization intensity became approximately zero at the Curie temperature of 120 ${ }^{\circ} \mathrm{C}$. As anti-oxidizing films, 20-nm-thick SiN films were fabricated on a glass substrate and those $50 \mathrm{~nm}$ thick were fabricated on the $\mathrm{Tb}_{22} \mathrm{Fe}_{78}$ film. These $\mathrm{SiN}$ films produced a localized mode in $a$-TbFe to provide a large rotation angle. ${ }^{(20)}$ The magnetic media structure was designed by the matrix approach $\operatorname{method}^{(21)}$ for localized light in magnetic media. The Kerr rotation angle was $0.7^{\circ}$, the Faraday rotation angle was $1.3^{\circ}$, the reflectivity was $8.8 \%$, and the transmissivity was $0.18 \%$ at $532 \mathrm{~nm}$.

Second, to control the hologram pattern, we fabricated an optical system using the tiling optical addressing method, which consists of a pulsed laser, a DMD, objective lenses, an $x-y-z$ stage, and magnetic media. The optical system for the tiling optical addressing method is shown in Fig. 1. A DMD (Discovery 1100, $1024 \times 768$ pixels, maximum driving speed of $22 \mathrm{kHz} /$ frame) was used for the tilling optical addressing method. The DMD showed part of the 2D hologram pattern. To write the hologram, a pulsed laser (Nd:YAG laser) operating at $355 \mathrm{~nm}$ with a pulse width of approximately 10 ns and a frequency of $10 \mathrm{~Hz}$ was used. The optical parametric oscillator was a MOPOSL-1P (Spectra-Physics) to control wavelength. The laser was used to illuminate the 
DMD. The DMD displayed the image of a $2 \mathrm{D}$ hologram pattern. The laser power ranged from 10 to $40 \mathrm{~mW}$ in $10 \mathrm{~mW}$ steps to fabricate the magnetic pixels. To expand the hologram area, the writing area was moved with an $x-y-z$ stage (the maximum moving distance was $20 \mathrm{~mm}$ with $1 \mu \mathrm{m}$ resolution).

The $2 \mathrm{D}$ hologram pattern on the DMD was transferred to the magnetic film by two object lenses whose focal lengths are 100 and $10 \mathrm{~mm}$. The pixel size was decreased to one-tenth of the original size of the DMD. Therefore, the pixel size of the DMD was $13.6 \mu \mathrm{m}$, and the magnetic pixel size was $1.36 \mu \mathrm{m}$. The viewing angle of the $3 \mathrm{D}$ images was about $23^{\circ}$ in accordance with eq. (1).

To demonstrate the $3 D$-MOSLM, we calculated a $2 \mathrm{D}$ hologram pattern of a $3 \mathrm{D}$ object by the computer-generated hologram method. The $2 \mathrm{D}$ hologram pattern was calculated by the half-zone plate method. ${ }^{(6,9)}$ This method has an advantages, that is, the calculation time is half that of the full-zone plate method and the reconstructed image can be cut from a conjugate image. The 3D object was a 3D framed-cube image, the length of a side of which was $3.74 \mathrm{~mm}$, the length of a side of the hologram area on the magnetic medium was $14 \mathrm{~mm}$, and the distance from the magnetic medium was $20 \mathrm{~mm}$ (Fig. 2). There were approximately $10000 \times 10000$ pixels in the hologram area.

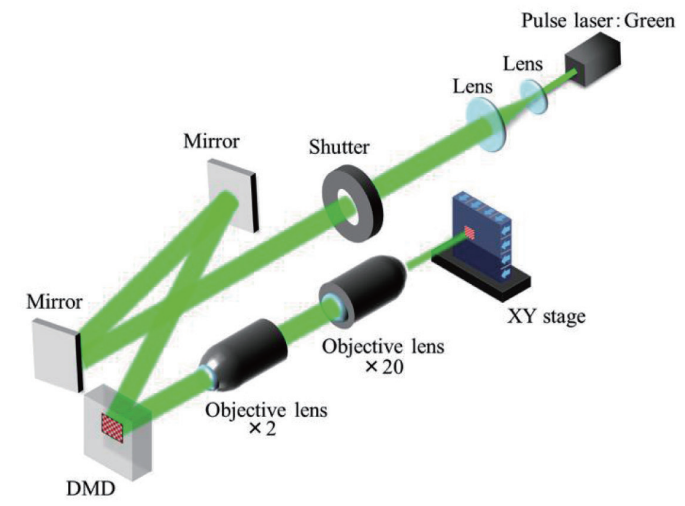

Fig. 1. (Color online) Optical system for the tilling optical addressing method.

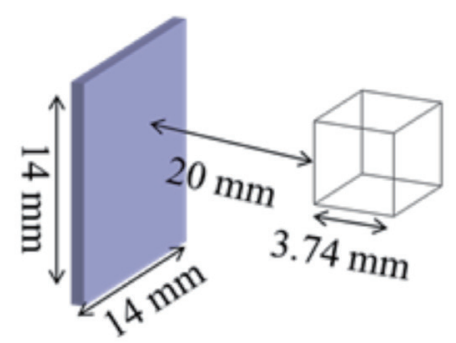

Fig. 2. (Color online) An original 3D image for the computer-generated hologram. 
We measured the characteristics of the holographic display of the $3 D$-MOSLM with a submicron magnetic pixel array. The optical system for object visualization consisted of a CW laser $\left(\lambda=532 \mathrm{~nm}, 8.6 \mathrm{~mW} / \mathrm{cm}^{2}\right)$ as the reference beam, a polarizer, the $3 D$-MOSLM with a magnetic hologram pattern, an analyzer, and a charge-coupled device (CCD) for the detection of 3D images. The polarization direction of the reconstructed image from the magnetic medium was rotated $90^{\circ}$ from the incident light. Therefore, this $3 D$-MOSLM had an adequate ratio of intensity of 1 st diffracted light to intensity of 0th transmitted light. ${ }^{(22)}$ The transmissivity of the polarizer and analyzer was about $35 \%$ and the extinction ratio was from $10^{-4}$ to $10^{-5}$. The diffraction ratio was $18 \%$ at $90^{\circ}$. This optical system operated in transmission mode. Therefore, the reconstructed image was represented as transmitted light from the $3 D$-MOSLM.

\section{Results}

Figure 3 shows the mesh pattern on the DMD and the mesh pattern of the magnetic pixels produced on the magnetic medium by the tiling optical addressing method. A square width was $136 \mu \mathrm{m}$ on the DMD, and a square was $10 \times 10$ pixels in Fig. 3(a). Figure 3(b) shows the magnetic pixel array when the laser power was $30 \mathrm{~mW}$. The hologram pattern of light on the DMD could be reduced to one-tenth its size and transferred to the magnetic film. In this result, a pixel $13.6 \mu \mathrm{m}$ in size on the DMD was reduced to $1.36 \mu \mathrm{m}$ on the magnetic pixels by objective lenses.

We wrote the magnetic pixel array of a $2 \mathrm{D}$ hologram pattern obtained by the computer-generated hologram.(6,9) Figure 4(a) is a part of the computer-generated hologram pattern that was displayed on the DMD. This image size was reduced to one-tenth of the original by the tiling optical addressing system and was transferred to the magnetic film. Figure 4(b) shows the transferred 2D hologram pattern. The 2D hologram pattern on the DMD was accurately transferred by reducing its size on the magnetic film.

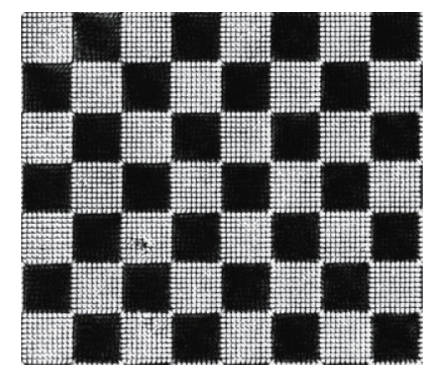

(a)

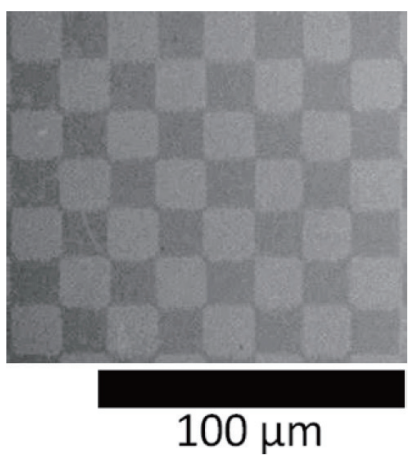

(b)

Fig. 3. Mesh pattern of magnetic pixels: (a) hologram pattern on the DMD and (b) magnetic pixel pattern on magnetic media. 


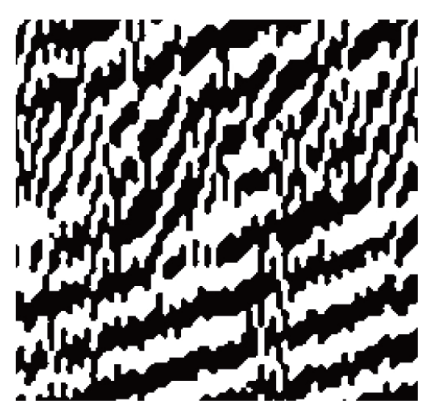

(a)

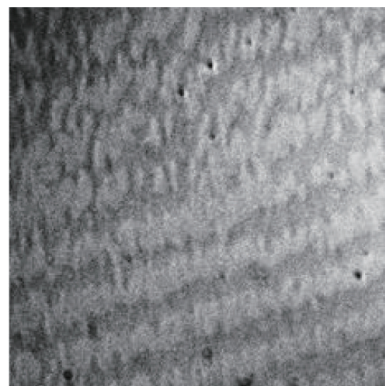

(b)

Fig. 4. Fringe pattern of 3D object: (a) part of the hologram image on the DMD and (b) magnetic pixel image of the same area.

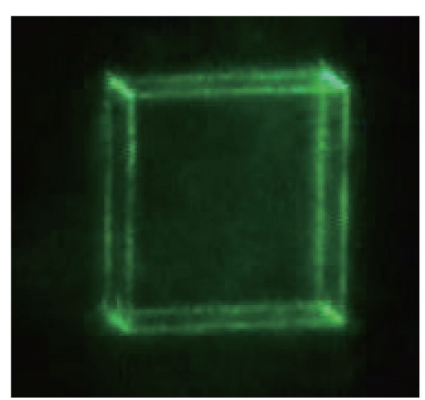

(a)

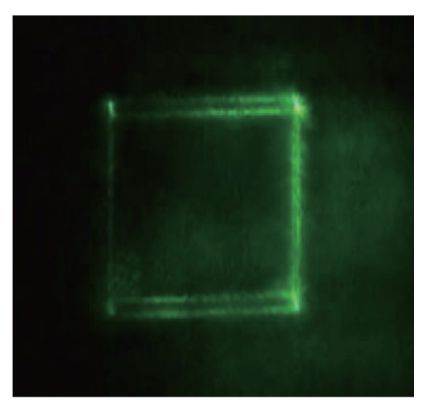

(b)

Fig. 5. (Color online) Reconstructed image of a 3D object: (a) viewing angle was $5^{\circ}$ from the left side and (b) viewing angle was $0^{\circ}$.

Figure 5 shows the reconstructed holographic 3D image. The 3D image could be reconstructed by transferring a $2 \mathrm{D}$ hologram pattern when a reference light illuminated the magnetic pixel array. The reconstructed images showed the same pattern that was designed by the computer. These results show that the $3 D$-MOSLM can represent a flexible 3D image.

\section{Conclusions}

A holographic display represents the image of a real 3D object. We have developed a wide-viewing-angle $3 D$-MOSLM. In this paper, we described the results of forming magnetic pixel patterns using the tilling optical addressing method. This optical system has a DMD to display any 2D hologram pattern, objective lenses to reduce the pixel size, and a pulsed laser for thermomagnetic recording. The magnetic pixel size was about 1.36 $\mu \mathrm{m}$. This optical method could represent any $3 \mathrm{D}$ image. 


\section{Acknowledgements}

This work was supported by a Grant-in-Aid for Young Scientists (B) 25820124.

\section{References}

1 D. Gabor: Nature 161 (1948) 777.

2 P. Hilaire, S. Benton and M. Lucente: J. Opt. Soc. Am. A 9 (1992) 1969.

3 F. Mok, J. Diep, H. K. Liu and D. Psaltis: Opt. Lett. 11 (1986) 748.

4 K. Maeno, N. Fukaya, O. Nishikawa and K. Sato: Proc. SPIE 2652 (1996) 13.

5 M. Stanley, R. Bannister, C. Cameron, S. Coomber, I. Cresswell, J. Hughes, V. Hui, P. Jackson, K. Milham, R. Miller, D. Payne, J. Quarrel, D. Scattergood, A. Smith, M. Smith, D. Tipton, P. Watsona and C. Slinger: Proc. SPIE 5005 (2003) 247.

6 Y. Takaki and Y. Tanemoto: Opt. Express 18 (2010) 10294.

7 T. Kozacki, G. Finke, P. Garbat, W. Zaperty and M. Kujawińska: Opt. Express 20 (2012) 27473.

8 Y. Z. Liu, X. N. Pang, S. Jiang and J. W. Dong: Opt. Express 21 (2013) 12068.

9 T. Mishina, F. Okano and I. Yuyama: Appl. Opt. 38 (1999) 3703.

10 D. Vettese: Nat. Photonics 4 (2010) 752.

11 L. J. Hornbeck: Proc. SPIE 3013 (1997) 27.

12 H. Takagi, K. Nakamura, T. Goto, P. B. Lim and M. Inoue: Opt. Lett. 39 (2014) 3344.

13 G. Fan, K. Pennington and J. H. Greiner: J. Appl. Phys. 30 (1969) 974.

14 R. S. Mezrich: Appl. Phys. Lett. 15 (1969) 132.

15 J. P. Waters: Appl. Phys. Lett. 9 (1966) 405.

16 J. H. Park, J. K. Cho, K. Nishimura, H. Uchida and M. Inoue: Phys. Status Solidi 201 (2004) 1976.

17 M. Komori, T. Numata, K. Tsutsumi, S. Inokuchi and Y. Sakurai: IEEE Trans. Magn. 20 (1984) 1042.

18 R. Sato, N. Saito and T. Morishita: IEEE Trans. Magn. 24 (1988) 2458.

19 Y. Martin, D. Rugar and H. K. Wickramasinghe: Appl. Phys. Lett. 52 (1988) 244.

20 M. Inoue, R. Fujikawa, A. Baryshev, A. Khanikaev, P. B. Lim, H. Uchida, O. Aktsipetrov, A. Fedyanin, T. Murzina and A. Granovsky: J. Phys. D 39 (2006) R151.

21 M. Inoue and T. Fujii: J. Appl. Phys. 81 (1997) 5659.

22 H. Haskal: IEEE Trans. Magn. 6 (1970) 542. 